\title{
PASTING INFINITE LATTICES
}

\author{
E. FRIED and G. GRÄTZER
}

(Received 29 September 1987)

Communicated by T. E. Hall

\begin{abstract}
In an earlier paper, we investigated for finite lattices a concept introduced by $\mathrm{A}$. Slavik: Let $A, B$, and $S$ be sublattices of the lattice $L, A \cap B=S, A \cup B=L$. Then $L$ pastes $A$ and $B$ together over $S$, if every amalgamation of $A$ and $B$ over $S$ contains $L$ as a sublattice. In this paper we extend this investigation to infinite lattices. We give several characterizations of pasting; one of them directly generalizes to the infinite case the characterization theorem of A. Day and J. Ježek. Our main result is that the variety of all modular lattices and the variety of all distributive lattices are closed under pasting.
\end{abstract}

1980 Mathematics subject classification (Amer. Math. Soc.) (1985 Revision): 06 B 05, 06 B 20.

\section{Introduction}

In [4], we investigate for finite lattices a concept introduced by A. Slavik [12] (see also G. Grätzer [6], Exercise 12 of Section V.4). Let $L$ be a lattice. Let $A, B$, and $S$ be sublattices of $L, A \cap B=S, A \cup B=L$. Then $L$ pastes $A$ and $B$ together over $S$, if every amalagamation of $A$ and $B$ over $S$ contains $L$ as a sublattice (A. Slavík used the term " $A$-decomposable".) Pasting generalizes the classical concept of gluing. see R. P. Dilworth and M. Hall [2].

A. Day and J. Ježek [1] proved the following theorem: let $\mathbf{V}$ be a nonmodular variety of lattices; if $\mathbf{V}$ is closed under the pasting of finite lattices, then $\mathbf{L}=\mathbf{V}$.

The research of both authors has been supported by the NSERC of Canada.

(C) 1989 Australian Mathematical Society 0263-6115/89 \$A2.00+0.00 
In [4], we answered a question raised in [1]: $N_{5}$ (the five-element nonmodular lattice) cannot be obtained from $M_{3}$ (the five-element modular nondistributive lattice) by pasting. In fact, the variety $\mathbf{M}$ of all modular lattices is closed under the pasting of finite lattices.

In this paper we extend the investigations of [4] to infinite lattices. In Section 2, we give several characterizations of pasting. One of them directly generalizes the characterization theorem of A. Day and J. Ježek [1] to the infinite case.

In Section 3, we investigate convex sublattices and ideal lattices of pasted lattices.

Section 4 contains the generalization of the main result from [4]: the variety $\mathbf{M}$ of all modular lattices is closed under pasting.

In Section 5 we prove that two distributive lattices pasted together yield a distributive lattice.

Section 6 contains some concluding comments.

The authors would like to express their appreciation to Ralph N. McKenzie, who patiently listened to a crude first draft of this paper, and to the members of the Lattice Theory and Universal Algebra Seminar in Winnipeg, who listened to the second draft of the paper. Their incisive comments and suggestions were much appreciated.

\section{Characterizations}

We start with a precise definition of pasting:

Definition 1. Let $L$ be a lattice. Let $A, B, S$ be sublattices of $L, A \cap B=S$, $A \cup B=L$. Let $f_{A}$ and $f_{B}$ be the embeddings of $A$ and $B$, respectively, into $L$. Then $L$ pastes $A$ and $B$ together over $S$, in notation, $L=\operatorname{Paste}(A, B, S)$, if whenever $g_{A}$ and $g_{B}$ are embeddings of $A$ and $B$ into a lattice $K$ satisfying $x g_{A}=x g_{B}$ for all $x \in S$, then there is a homomorphism $h$ of $L$ into $K$ satisfying $f_{A} h=g_{A}$ and $f_{B} h=g_{B}$ (see Figure 1 ).

Note that the homomorphism $h$ is always an embedding; this follows from the proofs of Theorems 5 and 6: we prove that $h$ is one-to-one.

To characterize pasting, we start with a simple lemma from B. Jónsson [11]:

Lemma 2. Let $A, B$, and $S$ be lattices, $A \cap B=S$. On $P=A \cup B$, we define a binary relation $\leq$ as follows:

(i) for $x, y \in A$ (and for $x, y \in B$ ), $x \leq y$ in $P$ if and only if $x \leq y$ in $A$ (respectively, $x \leq y$ in $B$ ); 


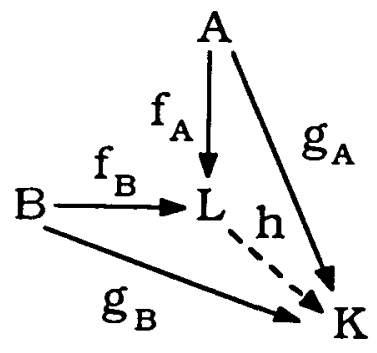

Figure 1

(ii) for $x \in A$ and for $y \in B, x \leq y$ in $P$ if and only if there exists an $s \in S$ with $x \leq s$ in $A$ and $s \leq y$ in $B$; and dually, for $y \leq x$.

Then $P$ is a poset $P(A, B, S)$. We shall use the notations $P$ and $P(A, B, S)$ interchangeably. The poset $P$ contains $A$ and $B$ as subposets.

If $L$ pastes $A$ and $B$ together over $S$, then $L$ as a poset is isomorphic to $P(A, B, S)$; the converse, however, is false in general. The poset $P(A, B, S)$ may be a lattice, but it may not be a pasting. There are many lattice constructions in the literature that put together lattices to obtain a new lattice. Two examples should suffice: the S-verklebte Summen of Ch. Hermann [9] and the hinged-product of E. Fried and G. Grätzer [3]. Most of these constructions put lattices together to form a poset; it is then proved that this poset is a lattice. It is important to remember that pasting is a lot more than $P(A, B, S)$ being a lattice.

Based on Lemma 2, we define $\wedge$ and $\vee$ as partial operations on $P$ :

Definition 3. Let $A, B$, and $S$ be lattices, $A \cap B=S$. On the set $P=A \cup B$, the partial algebra $\operatorname{Part}(A, B, S)$ with the partial binary operations $\wedge$ and $\vee$ is defined as follows:

(i) if $x \leq y$ in $P$, then $x \wedge y=x$ and $x \vee y=y$ in $\operatorname{Part}(A, B, S)$;

(ii) if $x, y, z \in A$ and $x \wedge y=z$ in $A$, then $x \wedge y=z$ in $\operatorname{Part}(A, B, S)$; and similarly for $x \vee y=z$ in $A$;

(iii) same as (ii), for $x, y, z \in B$.

Observe that, in general, $\operatorname{Part}(A, B, S)$ is not a partial lattice (or even a weak partial lattice) as defined in [6].

We now have everything we need to characterize pasting. However, for easier applicability in the proof in Section 4, we introduce an additional concept: 
Definition 4. Let $A, B$, and $S$ be lattices, $A \cap B=S$. Let $a \in A$ and $b \in B$. An $\{a, b\}$-sequence in $\operatorname{Part}(A, B, S)$ is a sequence of elements of $S: x_{1}, \ldots, x_{n}$ satisfying

(i) $x_{1} \leq \cdots \leq x_{n}$;

(ii ) $x_{1} \leq a$ (starts below $\left.a\right), x_{2} \leq b \vee x_{1}, x_{3} \leq a \vee x_{2}, \ldots, x_{2 k} \leq b \vee x_{2 k-1}$, $x_{2 k+1} \leq a \vee x_{2 k}, \ldots$; the target $t$ of this sequence is $a \vee x_{n}$, if $n$ is even (target above $a$ ), $b \vee x_{n}$, if $n$ is odd (target above $b$ ); or

(ii $\left.{ }_{b}\right) x_{1} \leq b$ (starts below $b$ ), $x_{2} \leq a \vee x_{1}, x_{3} \leq b \vee x_{2}, \ldots, x_{2 k} \leq a \vee x_{2 k-1}$, $x_{2 k+1} \leq b \vee x_{2 k}, \ldots$; the target $t$ of this sequence is $a \vee x_{n}$, if $n$ is odd (target above $a$ ), $b \vee x_{n}$, if $n$ is even (target above $b$ ).

For example, if $a \in S$, the singelton $a$ is an $\{a, b\}$-sequence (starts below $a)$ with target element $a \vee b$ (above $a)$. The sequence $x_{1}, x_{2}\left(x_{1}, x_{2} \in S\right.$ and $\left.x_{1} \leq x_{2}\right)$ with $x_{1} \leq a$ and $x_{2} \leq b \vee x_{1}$ is an $\{a, b\}$-sequence (starts below $a$ ) with target $a \vee x_{2}$ (above $a$ ).

The elements in ( $\mathrm{ii}_{a}$ ) form an increasing sequence in $A: a \leq a \vee x_{2} \leq \cdots \leq$ $a \vee x_{2 k} \leq \cdots$ and in $B: b \leq b \vee x_{1} \leq \cdots \leq b \vee x_{2 k+1} \leq \ldots$. The target is the last element of the first or the second sequence.

We describe pasting in terms of an order condition (Ord) and a sequence condition (Seq).

THEOREM 5. Let $L$ be a lattice. Let $A, B$, and $S$ be sublattices of $L, A \cap B$ $=S, A \cup B=L$. Then $L=\operatorname{Paste}(A, B, S)$ if and only if the following two conditions hold.

(Ord) For $a \in A$ and $b \in B$, if $a \leq b$, then there exists an $s \in S$ with $a \leq s$ in $A$ and $s \leq b$ in $B$, and dually for $b \leq a$.

(Seq) Let $a \in A-B$ and $b \in B-A$, and let $c=a \vee b$ in $L$. Then there exists an $\{a, b\}$-sequence with target $c$, and dually.

Another characterization of pasting is in terms of ideals:

Theorem 6. Let $L$ be a lattice. Let $A, B$, and $S$ be sublattices of $L, A \cap B$ $=S, A \cup B=L$. Then $L=\operatorname{Paste}(A, B, S)$ if and only if the following two conditions hold.

(Ord) For $a \in A$ and $b \in B$, if $a \leq b$, then there exists an $s \in S$ with $a \leq s$ in $A$ and $s \leq b$ in $B$, and dually for $b \leq a$.

(Id) If $X$ and $Y$ are ideals of $L$ satisfying $X-Y \subseteq A-B$ and $Y-X \subseteq B-A$, then $X \subseteq Y$ or $Y \subseteq X$; and dually, for dual ideals.

For finite lattices, in the presence of (Ord), (Id) takes on a simpler form:

$\left(\mathrm{Id}_{\text {fin }}\right)$ if $x, y \in L$ satisfy $(x \wedge y, x] \subseteq A-B$ and $(x \wedge y, y] \subseteq B-A$, then $x \leq y$ or $y \leq x$; and dually. 
Indeed, since $(x \wedge y, x] \subseteq(x]-(y],\left(\operatorname{Id}_{\text {fin }}\right)$ obviously implies (Id) for finite lattices. Conversely, if $L$ is finite and (Id) holds, then take $x, y \in L$ satisfying $(x \wedge y, x] \subseteq A-B$ and $(x \wedge y, y] \subseteq B-A$. We claim that $(x]-(y] \subseteq A-B$. Indeed, if $x \wedge y \in A-B$, then by (Ord), there exists a $s \in S$ with $x \wedge y<s$ and $s<y$, contradicting that $(x \wedge y, y] \subseteq B-A$. We get a similar contradiction if $x \wedge y \in B-A$. Finally, let $x \wedge y \in S$, and let $u \in(x]-(y]$; if $u \in B$, then $u \vee s \in B$, contradicting that $u \vee s \in(x \wedge y, x] \subseteq A-B$. Similarly, $(y]-(x] \subseteq B-A$. Thus (Id) applies, and we obtain $(x] \subseteq(y]$ or $(y] \subseteq(x]$, that is, $x \leq y$ or $y \leq x$, completing the proof of $\left(\operatorname{Id}_{\mathrm{fin}}\right)$.

Some of the steps in the proofs of Theorems 5 and 6 are implicit in A. Day and J. Ježek [1]. We give here complete proofs.

Crucial to the proofs is the definition of ideals in Part $(A, B, S)$ :

Definition 7. Let $A, B$, and $S$ be lattices, $A \cap B=S$, and $P=A \cup B$. An ideal $I$ of the partial algebra $\operatorname{Part}(A, B, S)$ is a subset of $P$ with the following two conditions:

(i) if $x \in I, y \in P$, and $y \leq x$, then $y \in I$;

(ii) if $x, y$, and $z \in I$ and $x \vee y=z$ in $\operatorname{Part}(A, B, S)$, then $z \in I$.

For a subset $X$ of $P,(X]$ will denote the smallest ideal containing $X$; if $X=\{x\}$, we write $(x]$ for $(X]$. An $A$-ideal $(B$-ideal $) I$ is of the form $I=(X]$, for some $X$ in $A$ (respectively, in $B$ ). The lattice of ideals will be denoted by $\operatorname{Id}(\operatorname{Part}(A, B, S))$. Dual ideals can be defined dually.

First, we need to know how to manipulate sequences and how sequences relate to ideals of $\operatorname{Part}(A, B, S)$.

Lemma 8. Let $A, B$ and $S$ be lattices, $A \cap B=S$, and $P=A \cup B$. Let $a \in A$ and $b \in B$. Then the following statements hold in $\operatorname{Part}(A, B, S)$.

(I) Let $x_{1}, \ldots, x_{n}$ be an $\{a, b\}$-sequence with target $t$. Then $x_{1}, \ldots, x_{n}, x_{n}$, $x_{n}, \ldots$ is again an $\{a, b\}$-sequence, if we add an even number of $x_{n}-s$, the target of the new sequence is $t$.

(II) If there exists an $\{a, b\}$-sequence that starts below $a$, then for every $\{a, b\}$-sequence with target $t$ there exists another $\{a, b\}$-sequence that starts below $a$ and has target $t$.

(III) Let $x_{1}, \ldots, x_{n}$ and $y_{1}, \ldots, y_{n}$ be $\{a, b\}$-sequences that start below $a$ with targets $t$ and $u$, respectively. Then $x_{1} \vee y_{1}, \ldots, x_{n} \vee y_{n}$ is an $\{a, b\}$-sequence that starts below a with target $t \vee u$.

(IV) Let $a \leq a^{\prime}$ and $b \leq b^{\prime}$, and let $x_{1}, \ldots, x_{n}$ be an $\{a, b\}$-sequence with target $t$ above $a$. Then $x_{1}, \ldots, x_{n}$ is an $\left\{a^{\prime}, b^{\prime}\right\}$-sequence with target $t \vee a^{\prime}$.

(V) We are given an $\{a, b\}$-sequence with target $t$ above $b$. Let $a^{\prime} \leq t$, $a^{\prime} \in A$. Then there exists an $\{a, b\}$-sequence with target $t^{\prime}$ above a such that $a^{\prime} \leq t^{\prime}$. 
(VI) Let $U$ be a subset of $P$. We define the set $U^{\text {Seq }}$ as follows: $z \in U^{\text {Seq }}$ if and only if there exist, $a, b \in P$ such that $a$ is a join of elements in $U \cap A$ and $b$ is a join of elements in $U \cap B$, and there exists an $\{a, b\}$-sequence with target $t$ satisfying $z \leq t$. Then $U^{\mathrm{Seq}}$ is an ideal of $\operatorname{Part}(A, B, S)$.

(VII) If in (VI), $U$ is contained in $U^{\mathrm{Seq}}$, then $U^{\mathrm{Seq}}$ is the ideal generated by $U$ in $\operatorname{Part}(A, B, S)$.

(VIII) Let us assume that any two elements of $P$ have a lower bound in $P$. Let $U$ be a subset of $L$ of the form $U_{A} \cup U_{B}$, where $U_{A}$ is a nonempty subset of $A-B$ and $U_{B}$ is a nonempty subset of $B-A$. Then $U^{\text {Seq }}$ is the ideal of $\operatorname{Part}(A, B, S)$ generated by $U$.

Proof. $A d$ (I). This is trivial

$A d$ (II). Let the $\{a, b\}$-sequence $x_{1}, \ldots, x_{n}$ with target $t$ be given; if it starts below $a$ we have nothing to prove. So let it start under $b$ (condition 4(ii ${ }_{b}$ ) applies): $x_{1} \leq b, x_{2} \leq a \vee x_{1}, \ldots$. Since there is an $\{a, b\}$-sequence starting below $a$, there is an element $s \in S$ with $s \leq a$. Define $x_{0}=x_{1} \wedge s$. For the sequence $x_{0}, x_{1}, \ldots, x_{n} 4(\mathrm{i})$ is obvious: $x_{0} \leq x_{1} \leq \cdots$. Moreover, $x_{0} \leq a$, $x_{1} \leq b \vee x_{0}(=b), x_{2} \leq a \vee x_{1}$ (by assumption), and so on, verifying 4(ii ${ }_{\mathrm{a}}$ ). Obviously, the target of the new sequence is unchanged.

$A d$ (III) and (IV). These are obvious.

$A d(\mathrm{~V})$. Let $x_{1}, \ldots, x_{n}$ be an $\{a, b\}$-sequence with target $t$ above $b$. Since $a^{\prime} \in A$ and $t \in B$, the assumption $a^{\prime} \leq t$ implies that there exists an $s \in S$ with $a^{\prime} \leq s \leq t=b \vee x_{n}$. Define $x_{n+1}=x_{n} \vee s$. Obviously, $x_{n} \leq x_{n+1}$ and $x_{n+1} \leq b \vee x_{n}$. Thus $x_{1}, \ldots, x_{n}, x_{n+1}$ is an $\{a, b\}$-sequence with target $a \vee x_{n+1}$ above $a$, and $a^{\prime} \leq a \vee x_{n+1}$, as claimed.

$A d$ (VI). Obviously, if $z \in U^{\text {Seq }}, w \in P$, and $w \leq z$, then $w \in U^{\text {Seq }}$. Now let $x, y \in U^{\text {Seq }}, x \vee y=z$ in $\operatorname{Part}(A, B, S)$; we want to show that $z \in U^{\text {Seq. If }}$ $x \vee y=z$ by virtue of $3(\mathrm{i}), z \in U^{\mathrm{Seq}}$ is trivial.

Let $x \vee y=z$ by virtue of 3 (ii), that is, $x, y, z \in A$ and $x \vee y=z$ in $A$. Since $x, y \in U^{\text {Seq }}$, we can choose $a_{1}, a_{2}$ that are joins of elements in $U \cap A$, and $b_{1}, b_{2}$ that are joins of elements in $U \cap B$, an $\left\{a_{1}, b_{1}\right\}$-sequence $x_{1}, \ldots, x_{n}$ with target $t_{1}$, an $\left\{a_{2}, b_{2}\right\}$-sequence $y_{1}, \ldots, y_{m}$ with target $t_{2}$, satisfying $x \leq t_{1}$ and $y \leq t_{2}$. With $a=a_{1} \vee a_{2}$ and $b=b_{1} \vee b_{2}, a$ is a join of elements in $U \cap A$ and $b$ is a join of elements in $U \cap B$; by (IV), both sequences are $\{a, b\}$-sequences with targets $t_{1}^{\prime} \geq t_{1}$ and $t_{2}^{\prime} \geq t_{2}$, establishing $x, y \in U^{\text {Seq }}$. So we can assume that $a_{1}=a_{2}=a$ and $b_{1}=b_{2}=b$.

If $t_{1}$ is the target of $x_{1}, \ldots, x_{n}$ above $b$, then by (V) (with $a^{\prime}=x$ ) we can change $x_{1}, \ldots, x_{n}$ to a sequence with target $t_{1}^{\prime}$ over $a, x \leq t_{1}^{\prime}$. Applying (V) again, if necessary, to the sequence $y_{1}, \ldots, y_{m}$, we conclude that we can assume that both sequences have targets over $a$. 
By (II), we can further assume that both sequences start below $a$ or both sequences start below $b$. By (I), therefore, we can assume that the two sequences have the same number of elements. Finally, by (III), we obtain an $\{a, b\}$-sequence with target $t_{1} \vee t_{2}$, establishing that $z=x \vee y \leq t_{1} \vee t_{2}$ is in $U^{\text {Seq. }}$.

If $x \vee y=z$ by virtue of 3(iii), we proceed by symmetry. Thus $U^{\text {Seq }}$ is an ideal.

$A d$ (VII). Let $W$ be an ideal containing $U$. An easy induction on $n$ shows that if we choose $a, b \in P$ such that $a$ is a join of elements in $U \cap A$ and $b$ is a join of elements in $U \cap B$, and an $\{a, b\}$-sequence $x_{1}, \ldots, x_{n}$ with target $t$, then $t \in W$, proving that $U^{\text {Seq }}=(U]$.

$A d$ (VIII). By (VII), it is enough to prove that for $a \in U_{A}$, we have $a \in U^{\text {Seq }}$ (and symmetricaly for $b \in U_{B}$ ). Choose an element $b \in U_{B}$. Let $c$ be a common lower bound of $a$ and $b$. There are two cases to consider.

FIRST CASE: $c \in A$. By Definition 3(i), there exists an $s \in S$ with $c \leq s \leq b$. The sequence $s$ can be regarded as an $\{a, b\}$-sequence that starts below $b$; its target is $a \vee s=a$ which majorizes $a$; thus $a \in U^{\text {Seq }}$.

SECOND CASE: $c \in B$. By Definition 3(i), there exists an $s \in S$ with $c \leq s \leq a$. The sequence $s, s$ can be regarded as an $\{a, b\}$-sequence that starts below $a$; its target is $a \vee s=a$ which majorizes $a$; thus $a \in U^{\text {Seq. This }}$ concludes the proof of (VIII), and therefore that of Lemma 8.

Now we prove Theorems 5 and 6 . Let $L=\operatorname{Paste}(A, B, S)$. We shall prove conditions (Ord), (Id), and (Seq) for $L$.

The MacNeille completion $P^{c}$ of $P=P(A, B, S)$ is a lattice containing $P$ as a subposet with the property that all joins and meets that exist in $P$ are preserved in $P^{c}$ (see, for example, [6]). By Definition $1, P$ is a sublattice of $P^{c}$, in fact, the sublattice $P=L$. Thus the partial ordering on $L$ is as described in Lemma 2, verifying condition (Ord) of Theorems 5 and 6.

Now let us take the lattice, $\operatorname{Id}(\operatorname{Part}(A, B, S))$, of all ideals of $\operatorname{Part}(A, B, S)$. The maps $a g_{A}=(a]$ for $a \in A$, and $b g_{B}=(b]$ for $b \in B$ embed $A$ and $B$, respectively, into $\operatorname{Id}(\operatorname{Part}(A, B, S))$, and they agree on $S$. By Definition 1 , there exists a homomorphism $h$ of $L$ into $\operatorname{Id}(\operatorname{Part}(A, B, S))$ extending $g_{A}$ and $g_{B}$.

Next we verify condition (Seq) of Theorem 5 . Let $a \in A-B$ and $b \in B-A$, and let $c=a \vee b$ in $L$. Without loss of generality, we can assume that $c \in A$. Then $c h=a h \vee b h$ in $\operatorname{Id}(\operatorname{Part}(A, B, S))$, that is, $(c]=(a] \vee(b]$. By (VIII) of Lemma $8\left(U_{A}=\{a\}\right.$ and $\left.U_{B}=\{b\}\right)$, there exists an $\{a, b\}$-sequence $x_{1}, \ldots, x_{n}$ with target $t$ satisfying $c \leq t$. Since $t \leq c$ obviously holds, we conclude that $t=c$, as claimed.

In the next step, we assume conditions (Ord) and (Seq) and we prove condition (Id) of Theorem 6. Let $X$ and $Y$ be ideals of $L$ satisfying $X-Y \subseteq$ 
$A-B$ and $Y-X \subseteq B-A$, and let $X \subseteq Y$ and $Y \subseteq X$ both fail. Let $U=(X-Y) \cup(Y-X)$; then (VIII) of Lemma 8 applies with $U_{A}=X-Y$ and $U_{B}=Y-X$; note that both $U_{A}$ and $U_{B}$ are closed under joins. Now take the ideal $U^{\text {Seq }}$ as defined in Lemma 8 . Choose any $a \in X-Y, b \in Y-X$, and an $\{a, b\}$-sequence $x_{1}, \ldots, x_{n}$ with target $t$. An easy induction on $i$ shows that $a \vee x_{i} \in X$ or $b \vee x_{i} \in Y$ holds for all $i$, hence $t \in X \cup Y$. Thus by (VI) of Lemma 8, $U^{\text {seq }}=X \cup Y$ is an ideal of $\operatorname{Part}(A, B, S)$.

By (Seq), for any $a \in X-Y$ and $b \in Y-X$, there exists an $\{a, b\}$-sequence with target $a \vee b$. Thus $a \vee b \in X \cup Y$, for example $a \vee b \in X$; but then $b \leq a \vee b \in X$ which contradicts that $b \in Y-X$. This proves (Id).

Now let $L$ be a lattice, let $A, B$, and $S$ be sublattices of $L, A \cap B=S, A \cup B=$ $L$. We have just proved that in the presence of condition (Ord), condition (Seq) implies condition (Id). We are now going to prove the converse.

So let us assume that conditions (Ord) and (Id) hold and let $a \in A-B$ and $b \in B-A$. Form the following ideals in $L$ :

$$
\begin{aligned}
& X_{0}=(a], \quad Y_{0}=(b), \\
& X_{i+1}=X_{i} \vee\left(Y_{i} \cap A\right], \quad i=1, \ldots, \quad Y_{i+1}=Y_{i} \vee\left(X_{i} \cap B\right], \quad i=1, \ldots
\end{aligned}
$$

Note that the ideal joins can be taken in $\operatorname{Id}(L)$ or $\operatorname{Id}(\operatorname{Part}(A, B, S))$; indeed, $X_{i}$ and $\left(Y_{i} \cap A\right]$ are both $A$-ideals, hence their join in $\operatorname{Id}(L)$ and in $\operatorname{Id}(\operatorname{Part}(A, B, S))$ is the same; similarly for $Y_{i}$ and $\left(X_{i} \cap B\right]$.

Let $X$ be the union of the $X_{i}, i=1, \ldots$, and let $Y$ be the union of the $Y_{i}$, $i=1, \ldots$. It is clear that $X \cup Y$ is the $\operatorname{Part}(A, B, S)$ ideal generated by $a$ and $b$.

Observe that $X-Y \subseteq A-B$. Indeed, if $x \in X-Y$ and $x \in B$, then $x \in X_{i}$ for some $i$, and hence $x \in Y_{i+1}$, contradicting that $x \in Y$ fails. Similarly, $Y-X \subseteq B-A$. Thus by (Id), $X \subseteq Y$ or $Y \subseteq X$, for example $X \subseteq Y$. Then $a \in Y$, and so $a \vee b \in Y$, that is, $a \vee b$ belongs to the $\operatorname{Part}(A, B, S)$ ideal generated by $a$ and $b$. It follows form Lemma 8(VIII) that $a \vee b$ is the target of some $\{a, b\}$-sequence, verifying (Seq).

To complete the proofs of Theorems 5 and 6, it remains to show that (Ord) and (Seq) imply that $L=\operatorname{Paste}(A, B, S)$. To prove this, by Definition 1 , we have to take a lattice $K$, and embeddings $g_{A}$ and $g_{B}$ of $A$ and $B$ into $K$ satisfying $x g_{A}=x g_{B}$ for all $x \in S$.

We define a map $h$ of $L$ into $K$ by $x h=x g_{A}$ for $x \in A$ and $x h=x g_{B}$ for $x \in B$. We have to show that $h$ is an embedding.

$h$ is isotone. Indeed, if $x \leq y$ and $x, y \in A$ or $B$, then $x h \leq y h$ since $h$ is isotone on $A$ and $B$. If, say, $x \in A-B$ and $y \in B-A$, then by (Ord), there is an $s \in S$ with $x \leq s \leq y$, and then $x h \leq s h$ and $s h \leq y h$ by the previous case, hence $h \leq y h$. 
$h$ is a homomorphism. Again, we can assume that $x \in A-B, y \in B-A$, and $x \vee y \in A$. By (Seq), there exists an $\{x, y\}$-sequence $x_{1}, \ldots, x_{n}$ with target $x \vee y$; without loss of generality we can assume that the sequence starts below $y$.

We proceed by induction on $n$. If $n=1$, then $x \vee y=x \vee x_{1}$, where $x_{1} \leq y$ and $x_{1} \in S$. Then

$$
\begin{aligned}
(x \vee y) h & =\left(x \vee x_{1}\right) h & & \\
& =x h \vee x_{1} h & & (\text { since } h \text { is an embedding of } A) \\
& \leq x h \vee y h & & \left(\text { since } x_{1} h \leq y h\right) .
\end{aligned}
$$

Since $h$ is isotone, the reverse inequality is trivial, $(x \vee y) h=x h \vee y h$.

Let $n>1$, and set $x^{\prime}=x \vee x_{1}$. If $x^{\prime} \in B$, then

$$
\begin{aligned}
(x \vee y) h & =\left(x^{\prime} \vee y\right) h & & \\
& =x^{\prime} h \vee y h & & \text { (since } h \text { is an embedding of } B) \\
& =x h \vee x_{1} h \vee y h & & (\text { since } h \text { is an embedding of } A) \\
& =x h \vee y h, & &
\end{aligned}
$$

as required. If $x^{\prime} \in A-B$, then $x_{2}, \ldots, x_{n}$ is an $\left\{x^{\prime}, y\right\}$-sequence which starts below $x^{\prime}$ with target $x^{\prime} \vee y$. Therefore,

$$
\begin{aligned}
(x \vee y) h & =\left(x^{\prime} \vee y\right) h & & \\
& =x^{\prime} h \vee y h & & \text { (by induction) } \\
& =x h \vee x_{1} h \vee y h & & \text { (since } h \text { is an embedding of } A) \\
& =x h \vee y h, & &
\end{aligned}
$$

completing the induction.

Using the dual argument, we establish that $(x \wedge y) h=x h \wedge y h$.

Finally, $h$ is one-to-one. In case $x<y$, we can use (Ord) to verify this. So we can assume that $x \in A-B, y \in B-A$, and, say, $x \vee y \in A$. If $x h=y h$, then using that $h$ is a homomorphism we obtain that

$$
x h=x h \vee y h=(x \vee y) h .
$$

Since $x<x \vee y, x h=(x \vee y) h$ contradicts that $h$ is one-to-one. This completes the proofs of Theorems 5 and 6.

In the finite case, in the presence of (Ord), condition (Id) is equivalent to the following condition:

(Cov) For $s \in S$, all the covers of $s$ in $L$ are in $A$ or all are in $B$; and dually. 
To verify this, we shall use the form ( $\left.\operatorname{Id}_{\text {fin }}\right)$ of (Id). Let (Cov) fail; then there is an $s \in S$ covered by $a$ in $A-B$ and $b$ in $B-A$. The elements $a$ and $b$ violate $\left(\operatorname{Id}_{\mathrm{fin}}\right)$ since

$$
(a \wedge b, a]=(s, a]=\{a\} \subseteq A-B
$$

and similarly for $(a \wedge b, b]$. Conversely, let $\left(\operatorname{Id}_{\mathrm{fin}}\right)$ fail with $a$ and $b$; then $a \wedge b \in S$ (if not, then say, $a \wedge b \in A-B$; by (Ord), there is an $s \in S$ with $a \wedge b<s<a$, contradicting that $(a \wedge b, b] \subseteq B-A)$ and the covers of $s$ are neither all in $A$ nor are all in $B$.

Thus we obtain a result of $A$. Day and J. Ježek [1]:

Corollary 9. Let $L$ be a finite lattice. Let $A, B$, and $S$ be sublattices of $L, A \cap B=S, A \cup B=L$. Then $L$ pastes $A$ and $B$ together over $S$ if and only if the conditions (Ord) and (Cov) hold.

\section{Some applications}

From the result of Day and Ježek (Corollary 9 above), in [4], we derived the result that if a finite lattice is pasted together, then the same holds for its intervals. Now that we have characterizations of pasting in the infinite case, it is natural to ask whether this result can be extended.

Corollary 10. Let the lattice $L$ paste the lattices $A$ and $B$ over $S$. Let $C$ be a convex sublattice of $L$ for which $C \cap S$ is not empty. Define $A_{1}=A \cap C$, $B_{1}=B \cap C, S_{1}=S \cap C$. Then $C$ pastes $A_{1}$ and $B_{1}$ over $S_{1}$.

Rather than proving this directly, we now introduce the concept of the rank; with it, we can quantify how the pasting of $A_{1}$ and $B_{1}$ is related to the pasting of $A$ and $B$.

Definition 11. Let $A, B$ and $S$ be lattices, $A \cap B=S, P=A \cup B$. For $x, y \in P$, we define the $\operatorname{rank}, \operatorname{rank}(x, y)$, of $x$ and $y$ in $\operatorname{Part}(A, B, S)$ as follows:

(i) if $x, y \in A$ or $x, y \in B$, then $\operatorname{rank}(x, y)$ is 0 ;

(ii) if there exists an $\{x, y\}$-sequence $x_{1}, \ldots, x_{n}$ with target $t$ satisfying $x, y \leq t$, then the smallest such $n$ is $\operatorname{rank}(x, y)$;

(iii) otherwise, $\operatorname{rank}(x, y)$ is undefined.

The following observation is useful: 
CoRollary 12. Under the conditions of Definition 11, for $x, y \in P$ and $s \in S$, if $\operatorname{rank}(x, y)$ is defined, then so is $\operatorname{rank}(x \vee s, y \vee s)$ and

$$
\operatorname{rank}(x \vee s, y \vee s) \leq \operatorname{rank}(x, y) \text {. }
$$

Proof. If the $\{x, y\}$-sequence $x_{1}, \ldots, x_{n}$ establishes that $\operatorname{rank}(x, y)=n$, then the $\{x \vee s, y \vee s\}$-sequence $x_{1} \vee s, \ldots, x_{n} \vee s$ establishes that

$$
\operatorname{rank}(x \vee s, y \vee s) \leq n \text {. }
$$

COROLlary 13. In Theorem 5, condition (Seq) can be replaced by the following condition:

(R) for every $x, y \in L, \operatorname{rank}(x, y)$ is defined, and dually.

Now we can state Corollary 10 in a stronger form:

Corollary 14. Let the lattice $L$ paste the lattices $A$ and $B$ over $S$. Let $C$ be a convex sublattice of $L$ for which $C \cap S$ is not empty. Define $A_{1}=$ $A \cap C, B_{1}=B \cap C, S_{1}=S \cap C$. Then for $x, y \in C$,

$$
\operatorname{rank}_{C}(x, y) \leq \operatorname{rank}_{L}(x, y)+1
$$

where $\operatorname{rank}_{C}(x, y)$ and $\operatorname{rank}_{L}(x, y)$ is the rank in $C$ and $L$, respectively. In particular, $\operatorname{rank}_{C}(x, y)$ is always defined.

Proof. If $x, y \in A$ or $B$, then $\operatorname{rank}_{C}(x, y)=\operatorname{rank}_{L}(x, y)=0$. Now let $\operatorname{rank}_{L}(x, y)=n>0$ be established by the $\{x, y\}$-sequence $x_{1}, \ldots, x_{n}$ in $L$. If $z=x \wedge y \in S$, then Corollary 12 (or its proof) shows that

$$
\operatorname{rank}_{C}(x, y) \leq \operatorname{rank}_{L}(x, y) .
$$

Now if $z \notin S$, then, say, $z \in A-B$. By (Ord), there is an $s \in S$ with $z<s \leq y$. If $x_{1}, \ldots, x_{n}$ is an $\{x, y\}$-sequence starting below $y$, then $x_{1} \vee s, \ldots, x_{n} \vee s$ is an $\{x, y\}$-sequence in $C$ establishing that

$$
\operatorname{rank}_{C}(x, y) \leq \operatorname{rank}_{L}(x, y) .
$$

Finally, if $x_{1}, \ldots, x_{n}$ is a $\{x, y\}$-sequence starting below $x$, then $s, x_{1} \vee s, \ldots$ is an $\{x, y\}$-sequence in $C$ establishing that

$$
\operatorname{rank}_{C}(x, y) \leq \operatorname{rank}_{L}(x, y)+1 .
$$

This completes the proof of Corollary 14.

The lattice of Figure 2 ( $S$ is represented by the black-filled, $A-S$ by the unfilled, and $B-S$ by the dot-filled elements) shows an example where

$$
\operatorname{rank}_{C}(x, y)=\operatorname{rank}_{L}(x, y)+1 .
$$




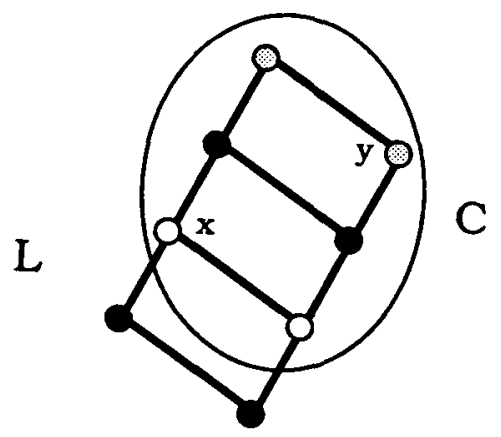

Figure 2

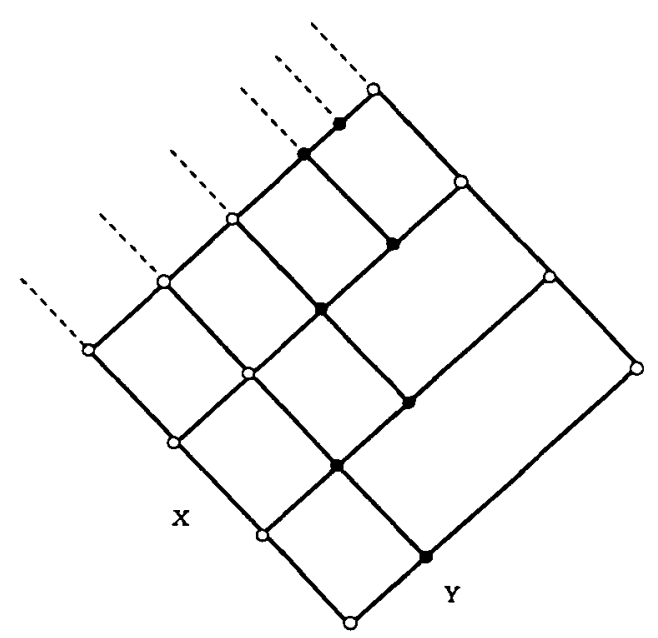

Figure 3

Thus the result of Corollary 14 is best possible.

In the infinite case we can pass from lattices to ideal lattices and ask whether from pasted lattices we get again pasted lattices.

Let $L=\operatorname{Paste}(A, B, S)$. Then $\operatorname{Id}(A)$, the ideal lattice of $A$, has a natural embedding into $L: \varphi_{A}: X \rightarrow(X]_{L}$.

Viewing $\operatorname{Id}(A)$ as a sublattice of $\operatorname{Id}(L)$, and similarly, $\operatorname{Id}(B)$ as a sublattice of $\operatorname{Id}(L)$, observe that $\operatorname{Id}(A) \cap \operatorname{Id}(B)=\operatorname{Id}(S)$. Indeed, if $I$ is an ideal of $L$ and $I \in \operatorname{Id}(A) \cap \operatorname{Id}(B)$, then for every $i \in I$ there is an $a \in A$ with $a \in I$ and $i \leq a$; so for this $a \in I$, there is an element $b \in b$ with $b \in I$ and $a \leq b$. By (Ord), there is an element $s \in S$ with $a \leq s \leq b$. Thus $i \leq s$ for some $s \in S$, proving that $I \in \operatorname{Id}(S)$. The converse is obvious. Finally, every ideal $I$ of $L$ 
is in $\operatorname{Id}(A)$ or $\operatorname{Id}(B)$. Indeed, if $I \notin \operatorname{Id}(A)$, then there is an $i \in I$ such that for all $j \in I$ with $i \leq j$, we have $j \in B$; similarly, if $I \notin \operatorname{Id}(B)$, then there is an $k \in I$ such that for all $j \in I$ with $k \leq j$ we have $j \in A$. But then $i \vee k \in I$ is in neither $A$ nor $B$, a contradiction.

Thus $\operatorname{Id}(L)=\operatorname{Id}(A) \cup \operatorname{Id}(B)$ and $\operatorname{Id}(A) \cap \operatorname{Id}(B)=\operatorname{Id}(S)$. It is logical to ask whether $\operatorname{Id}(L)$ pastes $\operatorname{Id}(A)$ and $\operatorname{Id}(B)$ together over $\operatorname{Id}(S)$.

LEMMA 15. Let $L$ be the lattice of Figure 3. Let $S$ be the sublattice of elements marked by solid dots; let $A$ be the sublattice of the elements of $S$ and the elements to the left; let $B$ be the sublattice of the elements of $S$ and the elements to the right. Then $L$ pastes $A$ and $B$ together over $S$ but $\operatorname{Id}(L)$ does not paste $\operatorname{Id}(A)$ and $\operatorname{Id}(B)$ together over $\operatorname{Id}(S)$.

Proof. Choose $X$ to be the left-axis and $Y$ the right-axis. We claim that $\operatorname{rank}(X, Y)$ in $\operatorname{Id}(L)$ is not defined. Indeed, let us form the ideals:

$$
\begin{aligned}
X_{0} & =X ; & Y_{0} & =Y ; \\
X_{i+1} & =X_{i} \vee\left(Y_{i} \cap A\right), \quad i=1, \ldots ; & Y_{i+1} & =Y_{i} \vee\left(X_{i} \cap B\right), \quad i=1, \ldots
\end{aligned}
$$

It is easy to see that $X_{i}$ is the ideal generated by $X$ and the $(2 i-1)$ st element of $S$, while $Y_{i}$ is generated by $Y$ and the $(i+1)$ st elements of $X$. If $\operatorname{rank}(X, Y)$ were defined, $\operatorname{rank}(X, Y)=n$, then either $X_{n}$ or $Y_{n}$ would equal $L$, a contradiction.

\section{Pasting modular lattices}

In this section we prove the following result, answering Problem 1 of [4]:

THEOREM 16. The variety $\mathbf{M}$ of all modular lattices is closed under pasting.

In the proof, we shall use the following notation:

Definition 17. For elements $x, y, z$ of the lattice $L, N_{5}(x, y \leq z)$ denotes that $x, y$, and $z$ satisfy the relations:

$$
y \leq z, \quad x \vee y=x \vee z, \quad x \wedge y=x \wedge z .
$$

If, in addition, $y<z$, then we write $N_{5}(x, y<z)$. If $N_{5}(x, y<3)$, then $x, y, z$ generate a sublattice isomorphic to $N_{5}$.

In the finite case, in [4], we proved Theorem 16 by way of contradiction: let $L$ be a finite nonmodular lattice, and let $L$ be the pasting of the modular sublattices $A$ and $B$ over $S$. We can choose $L$ as the smallest such lattice. 
Since $L$ is nonmodular, it contains elements $x, y, z$ satisfying $N_{5}(x, y<z)$. Clearly, $x \wedge z=0$ and $x \vee y=1$; indeed if $x \wedge z=u$ and $x \vee y=v$, and $0<u$ or $v<1$, then applying the Corollary 10 to $C=[u, v]$, we get a smaller lattice, contradicting the minimality of $L$. Thus $L$ is almost modular. no proper interval of $L$ can contain an $N_{5}$, hence the interval is modular. The proof heavily uses almost modularity.

In the general case there is no chance of finding a minimal or maximal $N_{5}$. Instead, we use the concept of the rank introduced in Section 3.

The proof of Theorem 16 relies heavily on the following two lemmas.

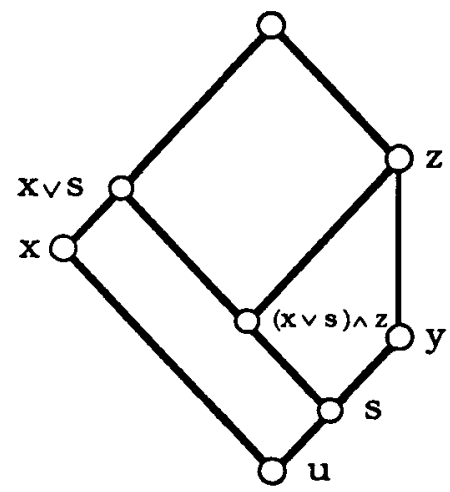

Figure 4

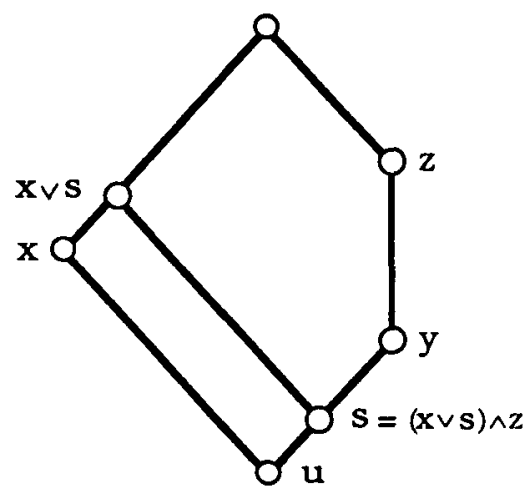

Figure 5

LEMMA 18. Let $L$ be a lattice, and let $x, y, z, s \in L$. If $N_{5}(x, y<z)$ and $x \wedge y \leq s<y$, then

(i) $N_{5}(x, s<(x \vee s) \wedge z)($ see Figure 4) or 
(ii) $s=(x \vee s) \wedge z$ and $N_{5}(x \vee s, y<z)($ see Figure 5).

Proof. Since $s \leq(x \vee s) \wedge z \leq x \vee s$, it follows that $x \vee s=x \vee((x \vee s) \wedge z)$; obviously, $x \wedge((x \vee s) \wedge z)=x \wedge s(=x \wedge z)$. Hence, $N_{5}(x, s \leq(x \vee s) \wedge z)$. Now (i) follows; (ii) is even easier.

LEMMA 19. Let $L$ be a lattice, and let $x, y, z, s \in L$. If $N_{5}(x, y<z)$ and $x \wedge y \leq s<x$, then

(i) $N_{5}(z, s<x \wedge(s \vee z))$ (see Figure 6)

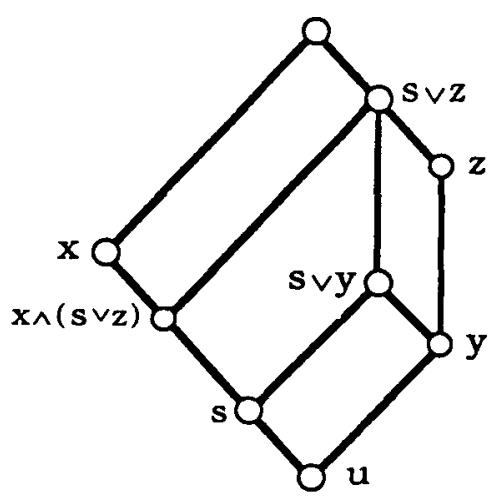

Figure 6

or

(ii) $s=x \wedge(s \vee z)$ and $N_{5}(x, s \vee y<s \vee z)$ (see Figure 7)

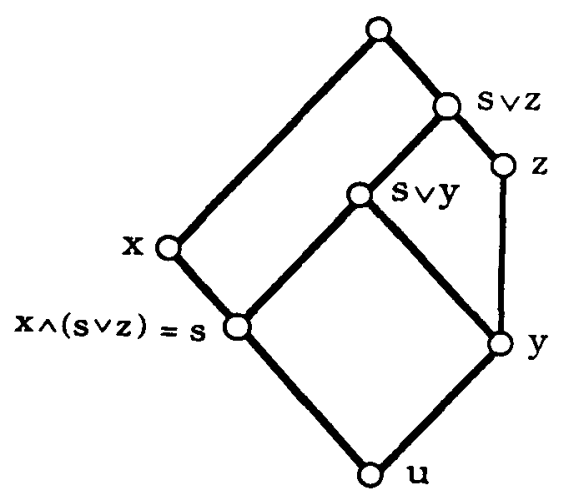

Figure 7

or 
(iii) $s=x \wedge(s \vee z), s \vee y=s \vee z$, and $N_{5}(s, y<z)$ (see Figure 8).

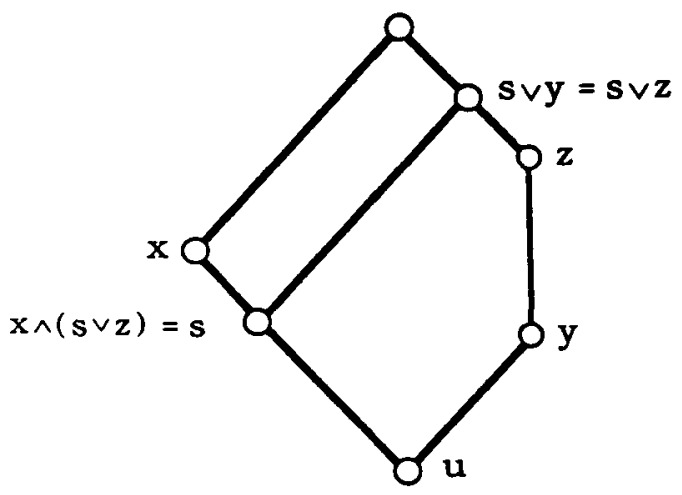

Figure 8

Proof. We prove (i) as 18(i) was proved; (ii) and (iii) are even easier.

Proof of Theorem 16. Let $L=\operatorname{Paste}(A, B, S)$. Let us assume that $A$ and $B$ are modular lattices, and $L$ is nonmodular. Since $L$ is nonmodular, it contains three elements $x, y, z$ satisfying $N_{5}(x, y<z)$; let $u=x \wedge z$ and $v=x \vee y$.

We shall consider several cases. As a rule, each case will be reduced to some previous cases.

Case 1. $x, y, z \in A$ or $x, y, z \in B$. Then the $N_{5}$ is in $A$ or $B$, contrary to the assumption that $A$ and $B$ are modular. So Case 1 cannot happen.

Case 2. $x \in S$. If $y, z \in A$ or $y, z \in B$, then Case 1 holds, a contradiction. By duality, we can assume that $y \in A-B$ and $z \in B-A$. By (Ord), there exists a $w \in S$ with $y<w<z$. Then, $N_{5}(x, y<w)$, contradicting Case 1 . Thus Case 2 cannot occur.

From now one, we can assume that $x \in A-B$ or that $x \in B-A$. Without loss of generality, we shall assume that $x \in A-B$.

Now there are only three possibilities: $y \in B-A$ and $z \in A ; y \in A$ and $z \in B-A ; y \in B-A$ and $z \in B-A$. We shall further classify by specifying where $u$ is: $A-B, B-A$, or $S$.

Case 3. $x \in A-B, y \in B-A, z \in A$. 
Case 3A. $u \in S$. We show that this case cannot occur by induction on $\operatorname{rank}(x, y)=n$. Let the $\{x, y\}$-sequence $p_{1}, \ldots, p_{n}(\in S)$ establish the rank.

Firstly, let the sequence start below $y$. Set $s=p_{1} \vee u$. Since $u \leq s<y$, by Lemma 18 , either $N_{5}(x, s<(x \vee s) \wedge z)$ which would contradict Case 1 since $x, s,(x \vee s) \wedge z \in A$, or $s=(x \vee s) \wedge z$ and $N_{5}(x \vee s, y<z)$ (see Figure $5)$. In the latter case, either $x \vee s \in S$, a contradiction by Case 2 , or $x \vee s \notin S$ and so $x \vee s \in A-B$ (since $x \vee s \in A$ ). Obviously, the sequence $p_{2}, \ldots, p_{n}$ establishes that $\operatorname{rank}(x \vee s, y) \leq n-1$, completing the discussion of the case.

Secondly, let the sequence start below $x$. Again, set $s=p_{1} \vee u$. Since $u \leq s<x$, by Lemma 19 , either

(i) $N_{5}(z, s<x \wedge(s \vee z)$ ) (see Figure 6), which would contradict Case 1 $(z, s, x \wedge(s \vee z) \in A)$,

or

(ii) $s=x \wedge(s \vee z)$ and $N_{5}(x, s \vee y<s \vee z)$ (see Figure 7); now if $s \vee y \in B-A$, then $N_{5}(x, s \vee y<s \vee z)$ satisfies the conditions of this case $(s \vee z \in A$ is obvious) and $\operatorname{rank}(x, s \vee y)<n$ (established by $\left.p_{2}, \ldots, p_{n}\right)$, and hence this is impossible by induction, or

(iii) $s=x \wedge(s \vee z), s \vee y=s \vee z$, and $N_{5}(s, y<z)$ (see Figure 8), a contradiction with Case 2.

Case 3B. $u \in A-B$. By (Ord), there exists an $s \in S$ with $u<s<y$. By Lemma 18, $N_{5}(x, s<(s \vee s) \wedge z$ ) (see Figure 4), which would contradict Case 1 since $x, s,(x \vee s) \wedge z \in A$, or $s=(x \vee s) \wedge z$ and $N_{5}(x \vee s, y<z)$ (see Figure $5)$, contradicting Case $3 \mathrm{~A}$.

CASE 3C. $u \in B-A$. this is impossible since $x, z \in A$, hence $x \wedge z=u \in A$.

Case $3^{\mathrm{D}}$. The dual of Case 3 . This case cannot hold by duality.

Case 4. $x \in A-B, y \in B-A, z \in B-A$.

Case 4A. $u \in S$. We show that this case cannot occur by induction on $\operatorname{rank}(x, y)=n$. Let the $\{x, y\}$-sequence $p_{1}, \ldots, p_{n}(\in S)$ establish the rank.

Firstly, let the sequence start below $y$. Set $s=p_{1} \vee u$. Since $u \leq s<y$, by Lemma 18, either

(i) $N_{5}(x, s<(x \vee s) \wedge z)$; now if $(x \vee s) \wedge z \in A$, then this contradicts Case $1(x, s,(x \vee s) \wedge z \in A)$; otherwise, $(x \vee s) \wedge z \subset B-A$ contradicting Case $3^{\mathrm{D}}$, or 
(ii) $s=(x \vee s) \wedge z$ and $N_{5}(x \vee s, y<z)$ (see Figure 5). Now if $x \vee s \in B$, then this contradicts Case $1(x \vee s, y, z \in B)$. Otherwise, obviously, $x \vee s \in A-B$, so $N_{5}(x \vee s, y<z)$ satisfies the conditions of Case $4 \mathrm{~A}$, and $p_{2}, \ldots, p_{n}$ establish that $\operatorname{rank}(x \vee s, y) \leq n-1$, completing the discussion of the case.

Secondly, let the sequence start below $x$. Again, set $s=p_{1} \vee u$. By Lemma 19, either

(i) $N_{5}(z, s<x \wedge(s \vee z)$ ) (see Figure 6); now if $x \wedge(s \vee z) \in B$, then we get a contradiction with Case $1(z, s, x \wedge(s \vee z) \in B)$; otherwise, $x \wedge(s \vee z) \in A-B$, and $N_{5}(z, s<x \wedge(s \vee z))$ is covered by Case 3 using the symmetry of $A$ and $B$; or

(ii) $s=x \wedge(s \vee z)$ and $N_{5}(x, s \vee y<s \vee z)$ (see Figure 7); now if $s \vee y$ or $s \vee z \in A$, then $N_{5}(x, s \vee y<s \vee z)$ is covered by a previous case; otherwise, $s \vee y, s \vee z \in B-A$, and so $N_{5}(x, s \vee y<s \vee z)$ satisfies the conditions of this case and rank $(x, s \vee y)<n$ (established by $p_{2}, \ldots, p_{n}$ ), and hence this is impossible by induction;

or

(iii) $s=x \wedge(s \vee z), s \vee y=s \vee z$, and $N_{5}(s, y<z)$ (see Figure 8), a contradiction with Case 2 .

Case 4B. $u \in A-B$. By (Ord), choose an $s \in S$ with $u<s<y$. By Lemma 18, $N_{5}(x, s<(x \vee s) \wedge z)$ (see Figure 4). Now if $(x \vee s) \wedge z \in A$, then this contradicts Case $1(x, s(x \vee s) \wedge z \in A)$; otherwise, $(x \vee s) \wedge z \in B-A$, covered by Case $3^{\mathrm{D}}$. Or $s=(x \vee s) \wedge z$ and $N_{5}(x \vee s, y<z)$ (see Figure 5). If $x \vee s \in S$, then we get to Case 2; otherwise, $x \vee s \in A-B$, and $(x \vee s) \wedge z \in S$, contradicting Case 4A.

Case 4C. $u \in B-A$. We show that this case cannot occur by induction on $\operatorname{rank}(x, y)=n$. Let the $\{x, y\}$-sequence $p_{1}, \ldots, p_{n}(\in S)$ establish the rank. By Lemma 19, either

(i) $N_{5}(z, s<x \wedge(s \vee z)$ ) (see Figure 6); if $x \wedge(s \vee z) \in B$, then we contradict Case $1(z, s<x \wedge(s \vee z) \in B)$; otherwise, $x \wedge(s \vee z) \in A-B$, and we contradict Case $3^{\mathrm{D}}$;

or

(ii) $s=x \wedge(s \vee z)$ and $N_{5}(x, s \vee y<s \vee z)$ (see Figure 7); if $s \vee y$ or $s \vee z \in A$, then $N_{5}(x, s \vee y<s \vee z)$ was covered in one of the previous cases (Cases 1 to $3^{\mathrm{D}}$ ); otherwise, $s \vee y, s \vee z \in B-A$, and $N_{5}(x, s \vee y<s \vee z)$ satisfies the conditions of Case $4 C$; obviously, the sequence $p_{2}, \ldots, p_{n}$ establishes that $\operatorname{rank}(x, s \vee y) \leq n-1$, completing the discussion of the case;

or 
(iii) $s=x \wedge(s \vee z), s \vee y=s \vee z$, and $N_{5}(s, y<z)$ (see Figure 8), which contradicts Case 2.

This completes the proof of the theorem.

\section{Distributive lattices}

In this section we prove

THEOREM 20. The variety $\mathbf{D}$ of all distributive lattices is closed under pasting.

Proof. Let $L=\operatorname{Paste}(A, B, S)$, where $A$ and $B$ are distributive lattices. By Theorem $16, L$ is modular.

If $L$ is not distributive, then it contains a sublattice $M_{3}$ (see Figure 9). Since $A$ and $B$ are distributive, we cannot have $x, y$, and $z \in A$ or $B$; without loss of generality we can assume that $x, y \in A$ and $z \in B-A$.

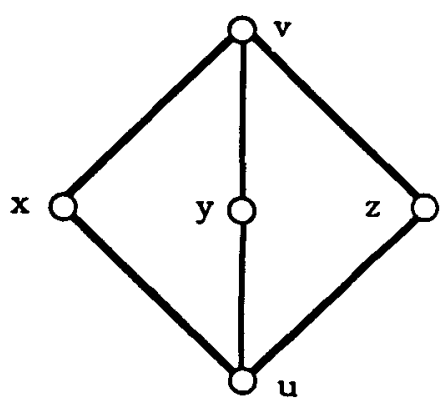

Figure 9

Claim A. $u \in S$ and there is no $s \in A$ with $u<s<z$.

Proof. Since $u=x \wedge y \in A$, if $u \notin S$, then $u \in A-B$. By condition (Ord) of Theorem 6, there is an $s \in S$ with $u<s<z$. To verify Claim A, it is sufficient to show that $u<s<z$ and $s \in A$ lead to a contradiction. Indeed, since $x, y$, and $s \in A$ and $A$ is distributive, we obtain that

$$
s=s \wedge(x \vee y)=(s \wedge x) \vee(s \wedge y)=u \vee u=u,
$$

a contradiction.

Claim B. $(z]-(y] \subseteq B-A$.

Proof. Let $p \in A$ satisfy $p \leq z$ but not $p \leq y$. Then $u<p \vee u \leq z$ and $p \vee u \in A$, contradicting Claim A. 
CLAIM C. $(y]-(z]$ contains an element of $B$.

Proof. If $(y]-(z] \subseteq A-B$, then by Claim $\mathrm{B},(y)$ and $(z]$ violate condition (Id) of Theorem 6.

Claim D. There is an $s \in S$ with $u<s<y$.

Proof. By Claim C, we can take a $p \in(y], p \notin(z]$, satisfying $p \in B$. Then $u<u \vee p \leq y$ and $u \vee p \in B$. By condition (Ord) of Theorem 6, there is an $s \in S$ with $u \vee p \leq s \leq y$.

Claim E. $L$ contains an $M_{3}^{\prime}=\left\{u_{1}, x_{1}, y_{1}, z_{1}, v_{1}\right\}$ with $x_{1} \in A-B, y_{1} \in S$, and $z_{1} \in B-A$.

Proof. Let $s$ be chosen as in Claim D. Let us define $x_{1}=(x \vee z) \wedge x$, $y_{1}=s, z_{1}=(s \vee x) \wedge z, u_{1}=x_{1} \wedge y_{1}$ and $v_{1}=x_{1} \vee y_{1}$. It is well known that these elements form a sublattice isomorphic to $M_{3}$.

Since $z_{1} \in[u, z], z_{1} \in B-A$ by Claim B. Also $y_{1} \in S$ by the choice of $s$. Finally, $x_{1} \in A-B$ because otherwise $M_{3}^{\prime} \subseteq B$, contradicting the distributivity of $B$.

Now we have the contradiction that proves the theorem. Indeed, by Claim B applied to $M_{3}^{\prime}$, we have $\left(z_{1}\right]-\left(y_{1}\right] \subseteq B-A$. By interchanging $A$ and $B$, Claim B applied to $M_{3}^{\prime}$ yields $\left(x_{1}\right]-\left(z_{1}\right] \subseteq A-B$. Hence the ideals $I=\left(x_{1}\right)$ and $J=\left(z_{1}\right]$ contradict condition (Id) of Theorem 6 .

\section{Concluding remarks}

In the proof of Theorems 5 and 6 , we only used that $g_{A}$ and $g_{B}$ were homomorphisms, except for the last step when we verified that $h$ was an embedding. This proves that the following is equivalent to Definition 1.

Definition $1^{\prime}$. Let $L$ be a lattice. Let $A, B, S$ be sublattices of $L, A \cap B=S$, $A \cup B=L$. Let $f_{A}$ and $f_{B}$ be the embeddings of $A$ and $B$, respectively, into $L$. Then $L$ pastes $A$ and $B$ together over $S$, in notation, $L=\operatorname{Paste}(A, B, S)$, if whenever $g_{A}$ and $g_{B}$ are homomorphisms of $A$ and $B$ into a lattice $K$ satisfying $x g_{A}=x g_{B}$ for all $x \in S$, then there is a homomorphism $h$ of $L$ into $K$ satisfying $f_{A} h=g_{A}$ and $f_{B} h=g_{B}$ (see Figure 1).

We can put this in another way: let $\varphi$ be a homomorphism of $L$ onto $L^{\prime}$; let $L$ paste $A$ and $B$ together over $S$, and let $A^{\prime}, B^{\prime}$, and $S^{\prime}$ be the images of $A, B, S$ under $\varphi$. Then $L^{\prime}$ pastes $A^{\prime}$ and $B^{\prime}$ together over $S^{\prime}$. 
Finally, we would like to mention an open problem:

Problem. Which varieties $\mathbf{V}$ of lattices are closed under pasting?

In this paper we have proved that $\mathbf{M}$ and $\mathbf{D}$ are such varieties. In [5], we have discussed continuumly many varieties of modular lattices (which are known to be closed under gluing) that are closed under the pasting of finite lattices. We do not know whether these same varieties are closed under pasting in general. More generally, it would be interesting to find out whether modular varieties of lattices that are closed under gluing are also closed under pasting.

\section{References}

[1] A. Day and J. Ježek, 'The amalgamation property for varieties of lattices', Mathematics Report \#1-83, Lakehead University.

[2] R. P. Dilworth and M. Hall, 'The embedding problem for modular lattices', Ann. of Math. (2) 45 (1944), 450-456.

[3] E. Fried and G. Grätzer, 'Notes on tolerance relations of lattices: On a conjecture of $\mathbf{R}$. McKenzie', manuscript; to appear in Acta Sci. Math. (Szeged).

[4] E. Fried and G. Grätzer, 'Pasting and modular lattices', manuscript (Abstract: Notices Amer. Math. Soc. 87T-06-209); to appear in Proc. Amer. Math. Soc.

[5] E. Fried, G. Grätzer, and H. Lakser, 'Projective geometries as cover preserving sublattices', manuscript (Abstract: Notices Amer. Math. Soc. 88T-06-47); to appear in Algebra Universalis.

[6] G. Grätzer, General lattice theory (Pure and Applied Mathematics Series, Academic Press, New York, 1978; Birkhäuser Verlag, Mathematische Reihe, Band 52, Basel, 1978).

[7] G. Grätzer, B. Jónsson, and H. Lakser, 'The Amalgamation Property in equational classes of modular lattices', Pacific J. Math. 45 (1973), 507-524.

[8] G. Grätzer and D. Kelly, 'Products of lattice varieties', Algebra Universalis 21 (1985), 33-45.

[9] Ch. Hermann, 'S-verklebte Summen von Verbänden', Math. Z. 130 (1973), 225-274.

[10] J. Ježek and A. Slavik, 'Primitive lattices', Czechoslovak Math. J. 29 (104) (1979), 595-634.

[11] B. Jónsson, 'Universal relation systems', Math. Scand. 4 (1956), 193-208.

[12] A. Slavik, 'A note on the amalgamation property in lattice varieties', Comment. Math. Univ. Carolinae 21 (1980), 473-478.

\section{Department of Mathematics}

University of Manitoba

Winnipeg, Manitoba, R3T 2N2

Canada 